\title{
O estágio curricular supervisionado em enfermagem sob a ótica dos concluintes do curso
}

\begin{abstract}
RESUMO | Objetivo: Investigar as dificuldades e contribuições do estágio supervisionado sob a ótica dos estudantes de enfermagem de uma instituição privada do município de Belém-Pa. Método: Estudo de caráter exploratório, de campo com abordagem qualitativa. A coleta de dados, por meio de entrevista semiestruturada ocorreu no mês de maio de 2019. A análise de dados foi de conteúdo segundo Bardin. Resultados: Evidenciaram-se uma divergência equilibrada entre os participantes, sobre o estágio como lugar de aprendizagem, visto que ao pensar sobre esse aspecto os estudantes associam a aprendizagem, ao relacionar teoria com a prática clínica e a realização de procedimentos técnicos específicos das atividades de enfermagem. Os estudantes identificam as dificuldades e as suas consequências para seu aprendizado e a contribuição do estágio para sua formação profissional. Conclusão: O estágio supervisionado é o momento de se desenvolverem competências e habilidades de ser enfermeiro tanto no aspecto assistencial, quanto gerencial.
\end{abstract}

Palavras-chaves: Estágio Clínico; Estudantes de Enfermagem; Metacognição.

\begin{abstract}
Objective: To investigate the difficulties and contributions of the supervised internship from the perspective of the nursing students of a private institution in the city of Belém-Pa. Method: Exploratory, field study with qualitative approach. Data collection through semi-structured interviews occurred in May 2019. Data analysis was based on Bardin's content. Results: A Balanced divergence was evidenced among the participants about the internship as A place of learning, since when thinking about this aspect, students associate learning, by linking theory with clinical practice and the realization of Specific Technical procedures of nursing activities. Students identify the difficulties and their consequences for their learning and the contribution of the internship to their professional training. Conclusion: The supervised internship is the moment to develop competencies and skills to be a nurse in both the care and managerial aspects.
\end{abstract}

Keywords: Clinical Clerkship; Students, Nursing; Metacognition.

RESUMEN | Objetivo: Investigar las dificultades y aportaciones de la pasantía supervisada desde la perspectiva de los estudiantes de enfermería de una institución privada en la ciudad de Belém-Pa. Método: Exploratorio, estudio de campo con enfoque cualitativo. La recopilación de datos a través de entrevistas semiestructuradas se produjo en mayo de 2019. El análisis de datos se basó en el contenido de Bardin. Resultados: Se evidenizó una divergencia equilibrada entre los participantes sobre la pasantía como lugar de aprendizaje, ya que al pensar en este aspecto, los estudiantes asocian el aprendizaje, vinculando la teoría con la práctica clínica y la realización de Procedimientos técnicos específicos de las actividades de enfermería. Los estudiantes identifican las dificultades y sus consecuencias para su aprendizaje y la contribución de las prácticas a su formación profesional. Conclusión: La pasantía supervisada es el momento de desarrollar competencias y habilidades para ser enfermera tanto en el aspecto asistencial como en el de gestión.

Palavras claves: Prácticas Clínicas; Estudiantes de Enfermería; Metacognición.

\section{Marcos Neuilly De Almeida Rodrigues}

Enfermeiro pelo Centro Universitário Metropolitano da Amazônia UNIFAMAZ. Belém-PA.

\section{Rosenil Brito Dos Santos}

Enfermeiro pelo Centro Universitário Metropolitano da Amazônia UNIFAMAZ. Belém-PA.

\section{Fernanda Farias Da Silva}

Enfermeira pelo Centro Universitário Metropolitano da Amazônia UNIFAMAZ. Belém-PA.

\section{Daniele Melo Sardinha}

Mestranda em Epidemiologia e Vigilância em Saúde, pelo Instituto Evandro Chagas IEC. Ananindeua-PA.

\section{Pedrinha do Socorro Castro dos Prazeres}

Acadêmica de Enfermagem pelo Centro Universitário Metropolitano da Amazônia UNIFAMAZ. Belém-PA.

\section{Ana Gracinda Ignácio Da Silva}

Doutora em Enfermagem, pela Universidade Federal do Rio de Janeiro UFRJ. Docente do Centro Universitário Metropolitano da Amazônia UNIFAMAZ. Belém-PA.

Recebido em: 20/07/2019

Aprovado em: 18/09/2019 INTRODUÇão

egundo a Resolução do Conselho
Federal de Enfermagem (COFEN),
número 441 de 2013, O (ECS) é o ato educativo supervisionado e obrigatório e desenvolvido no ambiente de trabalho visando à preparação dos estudantes para o trabalho. O estágio deve fazer parte do Projeto Político Pedagógico (PPP) do curso, que além de integrar o itinerário formativo do estudante, promove o aprendizado das características próprias da atividade profis- sional, objetivando o desenvolvimento deste para a vida cidadã e para o trabalho ${ }^{1}$.

O estágio supervisionado garante ao discente uma oportunidade de se autodescobrir como profissional, de conviver com outros colegas de profissão, de vivenciar habilidades como responsabilidades que Ihes são conferidas e liderança de equipe, tão essenciais para a formação do futuro enfermeiro ${ }^{2}$.

Essa atividade curricular é entendida por Dias et $\mathrm{al}^{3}$ como um importante instrumento para formação dos profissionais de enfermagem, no qual se desenvolvem habilidades profissionais e se aperfeiçoam técnicas e procedimentos realizados diariamente no exercício da profissão.

Esse período de aprendizagem em campo tem a função de consolidar o aprendizado teórico para formar profissionais mais capacitados e prepará-los para en- 
frentar o mercado de trabalho. Portanto, o estágio passa a ser valorizado no sentido de proporcionar o exercício de aproximação ao mundo real. Nessa perspectiva, explicita-se a preocupação com a formação de enfermeiros generalistas, ressaltando-se a obrigatoriedade do estágio curricular como elemento indispensável nessa construção ${ }^{4}$.

Porém, de um modo geral, a negociação para a realização do estágio na rede de saúde é tensionada pelo embate entre forças que envolvem o saber e o poder ${ }^{2}$. Além disso, vivencia-se no estágio supervisionado curricular dificuldades que vão desde técnicas, humanísticas, estruturais e de acessibilidade, levando a seguinte questão de pesquisa: Qual a ótica sobre o estágio curricular, dos concluintes do curso de bacharelado em enfermagem de uma Instituição Privada de Ensino Superior, do município de Belém?

O estudo é relevante pois, tem como base a percepção dos estudantes que realizam o estágio curricular, abordando sua experiencia durante esse período, estimulando-os a pensar sobre sua aprendizagem a partir desse espaço importante para significar o conteúdo teórico-prático no espaço de vida profissional real.

\section{MÉTODO}

Estudo exploratório, do tipo estudo de campo com abordagem qualitativa. A pesquisa foi realizada por meio de entrevista semiestruturada, a partir de um roteiro elaborada com base nos objetivos do estudo. Participaram do estudo 21 estudantes de ambos os gêneros, dos 43 alunos matriculados no último período do Curso de Graduação em Enfermagem de uma instituição de ensino superior privada do município de Belém-Pará. Essa amostra foi alcançada, considerando a facilidade de acesso aos participantes no campo de estágio ou na faculdade e disposição e aceitação deles para participar da pesquisa. Teve como critério de inclusão, alunos de ambos os sexos devidamente matriculados na Instituição de Ensino Superior, cursando o $10^{\circ}$ período do curso de bacharelado em enfermagem. Como critério de exclusão, alunos que não estejam em campos de estágio supervisionado durante a coleta de dados, ou que estejam de licença de saúde.

A coleta de dados aconteceu nos locais de estágio dos participantes e algumas na dependência da faculdade, considerava-se sempre a opção do participante. A entrevista foi registrada no roteiro de entrevista, pois os participantes preferiram que não fossem gravadas. Assim, procedeu-se a escuta sensível, registro e validação dos registros com os participantes.

Procedeu-se a análise de conteúdo de Bardin $^{5}$, para tal seguiu-se as fases de préanálise; exploração do material; tratamento dos resultados obtidos e interpretação. $\mathrm{Na}$ fase de pré-análise organizou-se todos os documentos de registros, por cada questão do roteiro e procedeu-se a análise exaustiva dos dados em busca de convergências e divergências nas informações produzidas que indicasse as categorias temáticas. Para tal foram construídos quadros e sistematizados nos mesmos as falas dos participantes em cada questão investigada.

Na segunda fase, a de exploração de material, foram identificadas as frases, palavras chaves, que após a leitura foram agrupadas e formaram as categorias temáticas do estudo. A partir das categorias temáticas foram agrupadas as falas que tinham o mesmo significado em quadros temáticos. Identificou-se cinco categorias temáticas: Estágio: oportunidade de aprendizagem; transferência de experiencia de aprendizagem; dificuldades no campo de estágio; contribuições do estágio supervisionado; estratégias de melhoria para o estágio supervisionado.

$\mathrm{Na}$ terceira fase, ocorreu o tratamento dos resultados e interpretação, realizou-se a descrição das categorias temáticas e a discussão dessas categorias, com base em autores que corroboraram os resultados encontrados.

A pesquisa atendeu aos preceitos éticos da Resolução 466/12 ${ }^{6}$ e foi submetida a avaliação ética e aprovada pelo Comitê de Ética em Pesquisa do Centro Universitário Metropolitano da Amazônia- UNIFAMAZ e aprovado sob protocolo: CAAE:09609419.8.0000.5701 e Número do Parecer: 3.222.290.

\section{RESULTADOS}

O quadro 1. Apresenta os dados demográficos dos participantes do estudo.

Dos 21 participantes, 18 tem idade entre 20 e 35 anos, a maioria (15) são mulheres, são solteiros (11) e casadas (os) ou em união estável (10). Portanto, tem-se um perfil de estudantes jovens, predominantemente feminina e dividido entre aqueles que já tem uma família constituída e os que não tem.

\section{Categorias Temáticas}

Os resultados sob a ótica dos estudantes quanto ao estágio supervisionado, foi distribuído em categorias temáticas que serão descritas a seguir.

Estágio: Oportunidade de aprendizagem

A metade dos participantes (11) consideram que ocorre aprendizagem no dia a dia do estágio, e os demais consideram que há pouca aprendizagem. A aprendizagem acon-

\section{Quadro 1 - CARACTERISTICAS DEMOGRÁFICAS DOS PARTICIPANTES}

$\begin{array}{llllll}\text { Idade } & \text { Qt } & \text { Gênero } & \text { Qt } & \text { Estado Civil } & \text { Qt } \\ \text { 20-30anos } & 14 & \text { MASC } & 06 & \text { CASADO } & 06 \\ \text { 31-40anos } & 06 & \text { FEM } & 15 & \text { UNIÃO ESTÁVEL } & 03 \\ +40 \text { anos } & 01 & - & - & \text { SOLTEIRO } & 11 \\ \end{array}$


tece sob a ótica desses estudantes, quando há uma relação entre teoria e prática, com oportunidade de realizar cuidados que foram aprendidos em sala de aula, conforme exemplificação de algumas falas:

“...oportunizando o agir, corrigir e melhorar algo que demonstramos dificuldades" $(4,2019)$.

"Consigo relacionar com qualificação o teórico da prática". (14, 2019)

O estágio também é visto como forma de ajudar a exercer a profissão dando oportunidades de desenvolver conhecimentos, condutas, aprimoramento de técnicas. A técnica utilizada para o desenvolvimento do estágio é considerada satisfatória, isso significa ter condições de relacionar teoria e prática e atender diversos casos clínicos, assim como, ter orientação adequada do preceptor e quando o preceptor apresenta autonomia no local de prática, a aprendizagem é desenvolvida com mais propriedade. É o que se verifica por exemplo, nas seguintes falas:

"Descrevo como plausível devido a forma de cobrança do preceptor por busca de conhecimento e aprimoramento de técnicas" $(7,2019)$.

"Somos orientados de que maneira devemos executar o que aprendemos em sala". $(4,2019)$

Nesse sentido, o estágio atendeu as expectativas dos estudantes de aprendizagem, de conseguir relacionar teoria e prática e realizar procedimentos. Alguns relataram que tinham receio do estágio por considerarem que ele poderia ser difícil, assim como, medo de não conseguirem colocar em prática a teoria. Como exemplifica-se com algumas falas:

"Percebi que a teoria e a prática se corresponderam" $(9,2019)$

"Por que os preceptores nos deram oportunidades para praticar." (4, 2019)

Entretanto, outra metade dos participantes caracterizam o estágio com pouco aprendizado, os que definem dessa forma afirmam que o fluxo de pacientes e atividades no turno da tarde é menor se comparado com a manhã. É relatado ainda, a falta de estrutura do local de estágio, assim como, pouca atenção por parte do preceptor e grande número de alunos por grupo. Além disso, nem sempre é possível relacionar teoria com a prática, e o tempo de estágio é considerado menor do que pensam que deveria ser. Como apontam as falas a seguir:

"Insatisfatório, quantidade grande de alunos para apenas um grupo" $(6,2019)$.

"De maneira falha, pois sem praticar o que aprendemos na teoria não teremos confiança e habilidades para exercer a profissão". (12, 2019).

Quanto as expectativas sobre o estágio supervisionado, uma significativa parcela dos participantes (13), relatou expectativas de que o estágio apresentasse mais oportunidades de aprendizagem, de realizar procedimentos técnicos, de atuar como enfermeiro na assistência e gerência do serviço, de mais disponibilidade do campo de prática, de certa autonomia nas consultas dos pacientes.

Entretanto, relatam poucas oportunidades para desenvolver atividades em virtude do horário (vespertino), poucos procedimentos, por falta de autonomia do preceptor no campo de estágio, principalmente, na atenção básica.

Observa-se que nesta categoria há uma divergência equilibrada entre os participantes, sobre o estágio como lugar de aprendizagem, visto que ao pensar sobre esse aspecto os estudantes associam a aprendizagem quando podem relacionar teoria com a prática clínica e realização de procedimentos técnicos específicos das atividades de enfermagem. Da mesma forma, definem a aprendizagem insatisfatória, quando as oportunidades de atendimento ao paciente e realização de técnicas são quantitativamente deficientes.

\section{Transferência de experiência de aprendizagem}

Nesta categoria, a maioria dos participantes (16) relataram que já levaram uma experiencia de aprendizagem para outra situação semelhante. Entre as experiencias que puderam reproduzir em mais de um momento de estágio estão: a consulta de enfermagem, pro- cedimentos como sondagens, curativos, ações educativas no pré-natal, acolhimento, coleta de PCCU, teste rápido para sífilis. Como observa-se nos seguintes relatos:

"Ações educativas de acolhimento, aleitamento materno etc." $(2,2019)$

"Tratamento de feridas em pé diabético e acompanhar a evolução dela. " $(12,2019)$

"Aspirar, passagem de sondas" $(20,2019)$

Essa é uma reflexão importante sobre seu aprendizado, pois o faz pensar no quanto ele se desenvolveu a partir do primeiro contato com uma determinada situação de aprendizagem e como ela ajudou quando se deparou com situações semelhantes.

\section{Dificuldades no campo de estágio}

Sobre esta categoria, a maioria dos participantes (13) relataram como dificuldades: distância dos locais de estágio, insegurança, de locomoção, infraestrutura inadequada dos locais de estágio, falta de equipamentos e materiais no campo de estágio, falta de logística para os alunos, falta de oportunidades para realizar atendimento e procedimentos como PCCU, falta de autonomia do preceptor.

Sobre a localização de campos de estágio, relatam que são em bairros considerados perigosos, de acesso difícil e distantes dos locais que residem. Esse fato faz com que prejudique o aprendizado, pois entre sair do local de trabalho ou de sua residência até o local de estágio, faz com que cheguem cansados, com sensação de fadiga física o que interfere no aproveitamento e aprendizagem. É o que se observa nos exemplos a seguir:

"Cansaço devido a distância que gerava fadiga, dificultando o aprendizado". $(17,2019)$

"Porque os campos são em bairros perigosos". $(1,2019)$

Quanto a infraestrutura dos locais de estágio, principalmente de atenção básica, a falta de equipamentos e materiais leva a improvisação, que alguns chamam de criatividade. Também a falta de um local destinado 
aos alunos, dificulta que eles se reúnam para discutir casos, tirar dúvidas. As dificuldades em relação ao atendimento, são indicadas pela baixa demanda de usuários ao setor no turno vespertino, assim como da pouca autonomia dos preceptores. Por exemplo:

"Poucos recursos para o trabalho" (16, 2019)

"População usa o serviço pela manhã." $(10,2019)$

Os estudantes identificam com clareza as dificuldades e suas consequências para seu aprendizado, demonstrando maturidade e preocupação com suas limitações. Indicam algumas alternativas, como por exemplo, procurar alocar os alunos em locais de estágio próximo a área em que residem, assim como, preceptores que tenham aproximação com os locais de prática, o que para eles pode diminuir algumas das dificuldades apontadas. Essa é uma problemática que reflete em primeiro lugar, a questão de segurança do estado e até nacional, entretanto, apesar de serem áreas consideradas "vermelhas" não se pode furtar de atender a população que nela vive, mas que sem dúvida é necessárias políticas públicas que garantam aos profissionais de saúde serenidade para esse atendimento e condições estruturais aos serviços de saúde.

Contribuições do estágio supervisionado

As contribuições do estágio supervisionado, percebidas pelos participantes do estudo são apontadas como aprimoração do conhecimento, segurança profissional, qualificação para o exercício profissional, crescimento, desenvolvimento e formação específica, gerando expectativa de se tornar um profissional qualificado e de contato com a realidade de saúde no Brasil, que de certa forma é o que vão lidar como profissionais.

Isso significa para os participantes, que o estágio supervisionado ajuda o estudante a se tornar um bom profissional, com ética e postura profissional. É no estágio que se aprende ser enfermeiro e como deve agir em diversas situações de conflitos e com o paciente, ou seja, ensina a tomar decisões assistenciais e gerenciais. Além disso, desenvolve a habilidade técnica fazendo com que eles se sintam com mais segurança para atuar como enfermeiro. É uma oportunidade de experiencia que auxilia no desenvolvimento da autonomia na realização de procedimentos, no contato direto com o paciente e na relação da teoria com a prática do sistema de saúde. Exemplificado nas falas a seguir:

"O estágio me deu a segurança de ser enfermeiro". $(3,2019)$

"A confiança que podemos adquirir em nós mesmos, sentir-se realmente enfermeiro". $(4,2019)$

Os que consideram fragilidades na contribuição do estágio supervisionado, indicam a falta de oportunidades, de logística e que não exerceram o gerenciamento e sim predominantemente, procedimentos técnicos, segundo esses, de responsabilidade mais do técnico de enfermagem, do que do enfermeiro. É importante ressaltar, entretanto, que os participantes reconhecem o seu papel nesse processo e refletem que precisam melhorar em várias áreas, que precisam ser mais participativos e ativos no seu aprendizado. Percebem que precisam aproveitar as oportunidades, saber sanar as dúvidas e se aprofundar nos estudos. Sentem-se que são responsáveis pelo seu aprendizado e que tem papel de fortalecer esse aprendizado para transferir isso no cuidado aos pacientes. Observa-se por exemplo nas falas:

"Bom aproveitamento das oportunidades, sanar dúvidas, aprofundamento nos estudos. " $(6,2019)$

"Temos o papel de fortalecer nosso aprendizado e procedimentos para passarmos para nossos pacientes de forma correta." $(19,2019)$

Quanto aos preceptores, os participantes de modo geral, vem esses profissionais como, bons profissionais, acessíveis, com conhecimentos teórico e prático, esforçados, dinâmicos e ativos, que mesmo com todas as dificuldades, se esforçam para melhorar o estágio. Isso quer dizer, que em sua maioria os participantes reconhecem o preceptor como alguém que repassam conhecimento e o direcionam em busca do aprendizado. Como exemplos a seguir:
"Bons profissionais, acessíveis, tem conhecimentos científicos, teórico e prático". $(6,2019)$

"Depende do preceptor, uns são muito esforçados e outros tem dificuldades" $(21,2019)$

A contribuição dos enfermeiros no seu aprendizado, é considerado pelos participantes importante, pois, são acolhedores na sua maioria, receptivos, cordiais, dão oportunidade para os estudantes realizarem as atividades, como por exemplo, consultas de enfermagem, porém são pouco participativos, não se envolvem no processo de aprendizagem, não se preocupam com os estudantes, o que consideram que isso ocorra pela sobrecarga de trabalho que eles têm na unidade. Como por exemplo:

"De muita importância pois ele é quem nos dá oportunidades para fazer consultas. " $(17,2019)$

"Pouco participativo no processo de aprendizagem, devido à sobrecarga de trabalho na unidade" $(7,2019)$

Dessa forma, os participantes sabem identificar a contribuição de cada segmento que faz parte do seu processo de aprendizagem no estágio curricular, inclusive seu próprio papel, com suas diferenças, possibilidades, limitações., o que indica um claro processo metacognitivos.

\section{Estratégias de melhoria para o estágio}

Os participantes relataram que algumas estratégias podem melhorar os campos de estágio. Entre essas, apontam: campos de estágio mais centrais, com melhor acessibilidade, de melhor qualidade de infraestrutura e material, ou seja, melhoria de convênios para prática e estágios curricular. Consideram ser necessário a reformulação nos campos de estágio, com mudanças de alguns desses locais, e considerar uma logística em relação aos campos, alocando os estudantes mais próximos de sua área residencial, consideram que tais medidas trariam impacto positivo ao aprendizado. Os participantes possuem uma visão crítica sobre os aspectos do estágio su- 
pervisionado que na sua percepção, precisam ser reorganizados e reformulados, para que os estudantes possam alcançar as expectativas que possuem em relação ao mesmo.

\section{DISCUSSÃO}

Os participantes (11) do estudo apontam, que no estágio supervisionado foi possível relacionar o conteúdo teórico com a prática e que o oportunizou o exercício profissional desenvolvendo conhecimentos, condutas, aprimoramento de técnicas, tal pensamento vai ao encontro das Diretrizes Curriculares Nacionais de Enfermagem DCN/ENF que apontam para a necessidade de definição de estratégias que articulem o saber (os conteúdos), o saber fazer (atitudes/habilidades) e o saber conviver (competências) e que esses parâmetros, por sua vez, oferecem os elementos para as bases filosóficas, conceituais e metodológicas que irão definir um perfil acadêmico e profissional com competências, habilidades e conteúdo, dentro de perspectivas e abordagens contemporâneas de formação, pertinentes e compatíveis com referências nacionais e internacionais, capazes de atuar com qualidade, eficiência e resolutividade, no SUS7.

O Estágio Curricular Supervisionado no Curso de Graduação em Enfermagem, aprovado pela Lei $n^{\circ}$. 11.788 de 25 de setembro de 2008 propõe desenvolver as atribuições conforme o exercício profissional, e determinar a realização do estágio como prática necessária para se alcançar o diploma (BRASIL, 2008). Portanto, a formação de um profissional para o mercado de trabalho não pode ser marcada apenas pela teoria é preciso, que o discente conheça seu espaço de atuação, e é no ECS, que se encontra a oportunidade de o aluno expandir conhecimentos, associando a teoria à prática ${ }^{2}$.

O momento esperado do estágio supervisionado gera expectativas, que nem sempre foram atendidas na percepção da maioria dos participantes, em relação ao desenvolvimento de procedimentos e atividades de competência do enfermeiro e da disponibilidade dos campos de estágio nem sempre positivas. Isso vai de encontro ao que Rigobello et $\mathrm{al}^{9}$ colocam sobre o ECS possibilitar, ampliar as oportunidades de desenvolvimento e aperfeiçoamento de competências, dentre as quais destaca-se a gerência, realizando práticas de enfermagem junto a indivíduos, famílias, grupos e comunidade, vivenciando, situações concretas do mundo do trabalho, interligadas às demandas da população e aos desafios e possibilidades dos serviços de saúde, o que possibilita o desenvolvimento de conhecimento, habilidades e atitudes no cenário de trabalho contribuindo para que o acadêmico de enfermagem desenvolva várias competências gerenciais e relacionadas a assistência, o que torna esse momento fundamental. . Além disso, a importância de se tomar a integralidade como objeto de reflexão de práticas da equipe de saúde e sua gerência se baseia na compreensão de que a construção da integralidade não ocorrerá em um lugar só, seja porque as várias tecnologias em saúde para melhorar e prolongar a vida estão distribuídas em ampla gama de serviços, seja porque a melhoria das condições de vida é tarefa para um esforço intersetorial ${ }^{10}$.

Apesar disso, a maioria dos participantes (16) indicam que já tiveram oportunidade de levar um aprendizado do estágio supervisionado, para outra situação, o que significa que a experiencia de aprendizagem foi positiva e que eles apresentam uma predisposição para aprendizagem de novas habilidades e competências. Isso ocorre devido a aprendizagem ser um processo psicológico que provoca mudanças no comportamento do indivíduo, que articula competências, habilidades e atitudes que resultam da interação dele com o meio onde está inserido, adquirindo a capacidade de utilizar o conhecimento na prática. Para tal, é necessário o processamento de informações que facilite a retenção, a recuperação e o uso posterior de novas informações, assim como, engloba comportamentos direcionados a aprendizagem e uso de novos conhecimentos e habilidades, bem como contribui para sua aplicação em diferentes contextos ${ }^{11}$.

Além disso, ao indicar aspectos de sua aprendizagem, e refletir sobre sua participação nesse processo, os participantes estão fazendo uso da cognição e da metacognição, pois tomam consciência do modo pelo qual o conhecimento é adquirido e gerenciam esse processo para facilitar o aprendizado e aplicá-lo em resolução de problemas a partir de sua interação com o meio ${ }^{12}$. Para que isso aconteça é preciso que utilizem estratégias de memorização, conexão de aprendizagem, elaboração e reestruturação do conhecimento ${ }^{8}$.

Entretanto, esses estudantes enfrentam dificuldades que vão desde o acesso ao local de estágio, a infraestrutura dos campos disponibilizados, deficiência de recursos, falta de oportunidades de desenvolvimento de habilidades, nem sempre são acolhidos de forma que esperam pelos enfermeiros e este não tem menor envolvimento em dividir sua experiencia e conhecimento com eles. Essas dificuldades são corroboradas por alguns autores, como Dias et $\mathrm{al}^{3}$, que apontam que a vivência no estágio nem sempre é positiva. Ao comunicar-se pela primeira vez com seu ambiente de estágio, os estudantes vivenciam diversos sentimentos em suas relações com o cliente, com o professor e com os próprios colegas da área da saúde, mobilizando diferentes expectativas. Dessa maneira, alguns acadêmicos sofrem muitas dificuldades para o ingresso no ECS, por inúmeros motivos, alguns por indisponibilidade de horários ou dificuldades de acesso, sendo que, alguns campos de estágios são situados em cidades diferentes da qual o aluno vive, e até mesmo por falta de local disponível para recebimento desses acadêmicos ${ }^{13}$. Sobre isso, Bosquetti e Braga ${ }^{14}$, ressaltam que, as dificuldades e angústias vivenciadas pelos graduandos no relacionamento com os pacientes, professor e com o ambiente em que estão inseridos produzem efeitos positivos e negativos no tocante as primeiras experiências práticas do aluno junto aos pacientes. Os fatores negativos estão relacionados aos cuidados de pacientes de alta complexidade, presenciar a morte, a percepção dos alunos de que os profissionais inseridos na área de saúde são insensíveis à dor e a morte.

Ao pensarem e perceberem a contribuição do estágio supervisionado em sua formação profissional, ressaltam que estão aprendendo a ser enfermeiros. Percebe-se que os estudantes sabem descrever e pontuar o que o estágio supervisionado oferece, desde o desenvolvimen- 
to de habilidades até o desenvolvimento de condutas comportamentais. Para isso, se utilizam do conhecimento metacognitivo, que se refere ao mundo de conhecimentos adquiridos relacionados com questões cognitivas ${ }^{15}$. Isso é importante, visto que a metacognição não é apenas um conhecimento sobre a cognição, mas uma etapa do processamento de nível elevado, que é adquirida e desenvolvida pela experiência e pelo conhecimento específico que é armazenado, é uma conquista evolutiva do ser humano, devido a sua necessidade de adaptação ao ambiente. ${ }^{16}$.

Observa-se que esses estudantes, sabem descrever seu aprendizado, ao longo do estágio supervisionado, relatam vivencias e seu significado, sua satisfação e insatisfação e isso se caracteriza em eventos metacognitivos, o que é corroborado no estudo de Silva e Peixoto $^{15}$, que pesquisaram sobre a aprendizagem do diagnóstico de enfermagem em formandos de enfermagem e evidenciaram que os acadêmicos pesquisados, também se utilizaram de eventos metacognitivos para descrever sobre seu processo de aprendizagem, compartilhando suas vivencias, experiencias, conhecimentos, dúvidas, satisfação e desapontamentos que fizeram parte de seu contexto de aprendizagem.

\section{CONCLUSÃO}

Para os participantes, o estágio supervisionado é caracterizado como um local onde devem aprender ser enfermeiros, e para a maioria dos participantes tem cumprido esse papel, entretanto, relatam também, que é um espaço onde enfrentam algumas dificuldades, que vão desde acesso ao local de estágio, infraestrutura deficiente e pouca demanda de pacientes para o atendimento, principalmente, na atenção básica. Os estudantes apontam que o estágio supervisionado, atendeu as expectativas em parte e que a atuação do preceptor foi importante em alguns casos, porém, insuficiente em outros momentos por falta de autonomia e pela ausência de um planejamento conjunto com os enfermeiros das unidades. Sobre os enfermeiros da unidade, os participantes definem como totalmente omissos no processo de aprendizagem dos alunos, limitando-se ao acolhimento e colocando à disposição a unidade. Consideram que algumas medidas podem contribuir para a melhoria do estágio supervisionado, como por exemplo, melhorar a acessibilidade aos campos de estágio, convênios com campos que sejam mais bem estruturados para receberem os alunos e qualificação da preceptoria. Conclui-se, que sob a ótica dos estudantes, o estágio supervisionado é o momento de desenvolverem as competências e habilidades de ser enfermeiro tanto no aspecto assistencial, quanto gerencial, porém, que atende em parte a esse propósito. Observa-se, portanto, a necessidade de discussões sobre estratégias que viabilizem o melhor aproveitamento pelos discentes desse momento, que se configura, entre outras estratégias, na maior aproximação com os campos de prática e assegurar que os estudantes desenvolverão as competências exigidas para sua futura prática profissional.

\section{Referências}

1. Marchioro D, Ceratto P, Bittencourt JVV, Martini JG, Silva CC da, Silva TG Da. Estágio Curricular Supervisionado: Relato Dos Desafios Encontrados Pelos (As) Estudantes. Arq Ciênc Saúde UNIPAR. 2017;21(2):119-22.

2. Lima D, Pereira O. Contribuições Do Estágio Supervisionado Para a Formação Do Profissional De Enfermagem: Expectativas E Desafios. Rev Enferm Contemp. 2014;3(2).

3. Dias EP, Stutz BL, Resende TC de, Batista NB, Sene SS de. Expectativas de alunos de enfermagem frente ao primeiro estágio em instituições de saúde. Rev Psicopedag. 2014;31(94):44-55.

4. Martins KRM, Oliveira T de, Filho ALDB, Gouveia PS, Sousa, Almeida EP de 0 , et al. Perspectiva de acadêmicos de enfermagem diante dos estágios supervisionados. C\&D-Revista Eletrônica da Faionar. 2016;9(1):56-73.

5. Bardin L. Análise de Contéudo. 70th ed. Pinheiro A, editor. São Paulo; 2016.281 p. 6. Brasil M da S. Resolução No 466, de 12 de dezembro de 2012 [Internet]. Diário Oficial da União DOU. 2012 [cited 2019 Sep 14]. p. 12. Available from: https://conselho.saude.gov.br/resolucoes/2012/Reso466.pdf

7. Teixeira E. 0 ensino de graduação em enfermagem no Brasil : o ontem, 0 hoje, o amanhã [Internet]. Brasília: INEP, Ministério da Educação; 2006 [cited 2019 Jul 6]. 69-96 p. Available from: https://www.estantevirtual.com.br/ boniek/elizabeth-teixeira-euclea-gomes-e-outras-ensino-de-graduacao-em-enfermagem-no-brasil-o-ontem-o-hoje-e-o-265763692?gclid=Cj0KCQjwjYHpBRC4ARIsAI-3GkHXI2BvDJ7TnLy5IXHD4z9HOOmBmxrN3IBQ-XB1WAbUIZjgAaP-xeAaAs_DEALw_wcB

8. Brasil M da E. LEI No 11.788, DE 25 DE SETEMBRO DE 2008. [Internet]. Diário Oficial da União DOU. 2008 [cited 2019 Sep 14]. Available from: http:// www.planalto.gov.br/ccivil_03/_ato2007-2010/2008/lei/111788.htm 9. Rigobello JL, Bernardes A, Moura AA de, Zanetti ACB, Spiri WC, Gabriel $C S$, et al. Supervised Curricular Internship and the development of management skills: a perception of graduates, undergraduates, and professors. Esc Anna Nery [Internet]. 2018 Apr 9 [cited 2019 Sep 14];22(2).
Available from: http://www.scielo.br/scielo.php?script=sci_arttext\&pi$d=S 1414-81452018000200203 \& \operatorname{lng}=e n \&$ tIng=en

10. Fracolli LA, Zoboli ELP, Granja GF, Ermel RC. Conceito e prática da integralidade na Atenção Básica: a percepção das enfermeiras. Rev da Esc Enferm da USP [Internet]. 2011 Oct [cited 2019 Sep 14];45(5):1135-41. Available from: http://www.scielo.br/scielo.php?script=sci_arttext\&pi$\mathrm{d}=\mathrm{S} 0080-62342011000500015 \& \mathrm{lng}=\mathrm{pt} \& \mathrm{t}$ lng $=\mathrm{pt}$

11. Coelho Junior FA, Borges-Andrade JE. Uso do conceito de aprendizagem em estudos relacionados ao trabalho e organizações. Paid (Ribeirão Preto) [Internet]. 2008 [cited 2019 Jul 6];18(40):221-34. Available from: http://www.scielo.br/scielo.php?script=sci_arttext\&pi$d=S 0103-863 \times 2008000200002 \& \operatorname{lng}=p t \&$ tlng $=p t$

12. Neves DA de B. Meta-aprendizagem e Ciência da Informação: uma reflexão sobre 0 ato de aprender a aprender. Perspect em Ciência da Informação [Internet]. 2007 Dec [cited 2019 Jul 6];12(3):116-28. Available from: http://www.scielo.br/scielo.php?script=sci_arttext\&pi$\mathrm{d}=$ S1413-99362007000300009\&/ng=pt\&tlng=pt

13. Silva CMV da, Silva LF da, Silva MEBV da S, França SB de, Silva ARS. Sentimentos dos enfermeiros frente ao estágio curricular: Quais as dificuldades e expectativas? Cad Grad. 2013;1(1):51-66.

14. Bosquetti LS, Braga EM. Reações comunicativas dos alunos de enfermagem frente ao primeiro estágio curricular. Rev da Esc Enferm da USP [Internet]. 2008 Dec [cited 2019 Sep 14];42(4):690-6. Available from: http://www.scielo.br/scielo.php?script=sci_arttext\&pi$d=50080-62342008000400011$ \&lng $=$ pt\&tlng $=p t$

15. Silva AGI da, Peixoto MAP. Metacognição e aprendizagem do diagnóstico de enfermagem Experiencias e perspectivas. 1st ed. Novas Edições Acadêmicas; $2015.60 \mathrm{p}$.

16. Andretta I, Silva JG da, Susin N, Freire SD. Metacognição e Aprendizagem: como se relacionam? PSICO. 2010;41(1):7-13. 